\title{
Quelles évolutions possibles pour les systèmes de polyculture-élevage? Résultats d'ateliers participatifs et de modélisation dans quatre régions françaises
}

\author{
Claire Mosnier ${ }^{1, *}$, Nelly Dubosc ${ }^{2}$, Ikram Abdouttalib ${ }^{1}$, Dominique Candau ${ }^{3}$, Yannick Carel $^{4}$, \\ Sophie Chauvat $^{5}$, Florian Fougy ${ }^{6}$, Émilie Guerre ${ }^{7}$, Lionel Magnin ${ }^{8}$ et Sonia Ramonteu ${ }^{9}$ \\ ${ }^{1}$ Université Clermont-Auvergne, INRAE, VetAgro Sup, UMR 1213 Herbivores, 63122 Saint-Genès-Champanelle, France \\ 2 Chambre régionale d'agriculture Occitanie, 31320 Auzeville-Tolosane, France \\ ${ }^{3}$ Chambre départementale d'agriculture des Vosges, 88026 Épinal, France \\ 4 ARVALIS - Institut du végétal, 45000 Orléans, France \\ 5 IDELE - Institut de l'élevage, Supagro, 34060 Montpellier, France \\ ${ }^{6}$ Chambre régionale d'agriculture de Normandie, 14000 Caen, France \\ 7 Chambre départementale d'agriculture de la Meuse, 55000 Savonnières-devant-Bar, France \\ ${ }^{8}$ FR CIVAM Pays de la Loire, 44390 Saffré, France \\ 9 ACTA, 75595 Paris, France
}

\begin{abstract}
Résumé - Les systèmes de polyculture-élevage peuvent utiliser les complémentarités entre les cultures et l'élevage pour réduire leur consommation d'intrants. Dans quatre régions françaises, des réunions d'experts ont été organisées pour discuter de l'évolution possible de ces systèmes selon trois scénarios prospectifs contrastés : «Ultra-libéralisation et mondialisation galopante» (S1), «Économie territoriale et recentrage sur la qualité» (S2) et "Transition agroécologique et énergétique » (S3) et pour réfléchir aux modalités possibles pour soutenir la polyculture-élevage dans chacun des scénarios. Pour appuyer ces discussions, les impacts des éléments structurants des scénarios ont été simulés à l'aide du modèle bioéconomique Orfee pour une ferme type de culture-élevage de chaque région. Les experts s'accordent pour dire que le S1 irait vers une réduction de l'élevage et une plus grande spécialisation en grandes cultures, que le S2 serait le plus favorable à des systèmes diversifiés et que le S3 induirait une baisse de l'élevage, mais une intégration plus forte entre élevage et cultures. Les résultats des simulations vont dans le même sens, excepté pour la production laitière qui augmente dans le $\mathrm{S} 1$ en raison de la prévision d'une augmentation du prix du lait et d'une main-d'œuvre non limitante. Les simulations ont permis de calculer des indicateurs de durabilité : S1 est supérieur pour les indicateurs économiques, S2 meilleur pour les indicateurs sociaux et S3 plus favorable à l'environnement. Les simulations soulignent également la nécessité de maintenir l'élevage dans une transition agroécologique pour réduire les engrais minéraux et les pesticides.
\end{abstract}

Mots clés : polyculture-élevage / prospective / approche participative / modèle bioéconomique

\begin{abstract}
What are the possible developments for polyculture-livestock systems? Results of participatory and modelling workshops in four French regions. Crop-livestock systems can use complementarities between crops and livestock to reduce their input consumption. In four French regions, expert meetings were organized to discuss the possible evolution of these systems according to three contrasting prospective scenarios: "Ultra-liberalization and globalization" (S1), "Territorial economy and refocusing on quality" (S2) and "Agro-ecological and energy transition" (S3), and to reflect on possible modalities to support crop-livestock systems in each of the scenarios. To support these discussions, the impacts of the structuring elements of the scenarios were simulated using the Orfee bio-economic model for a typical crop-livestock farm in each region. The experts agreed that S1 would lead to a reduction in livestock production and greater specialization in field crops, S2 would be the most favourable to diversified systems and S3 would lead to a reduction in livestock production, but with greater integration between
\end{abstract}

\footnotetext{
* Auteur de correspondance : claire.mosnier@inrae.fr
} 
livestock and crops. The results of the simulations go in the same direction, except for milk production, which increases in S1 due to the forecast increase in milk prices and non-limiting labour force. The simulations were used to calculate sustainability indicators: S1 is better for economic indicators, S2 better for social indicators and S3 more favourable to the environment. The simulations also highlight the need to maintain livestock farming in an agro-ecological transition to reduce mineral fertilizers and pesticides.

Keywords: crop-livestock farms / prospective / participatory approach / bio-economic model

\section{Introduction}

Les systèmes de polyculture-élevage font historiquement partie intégrante du paysage agricole français. Ces systèmes étaient dominants en France jusque dans les années 1950 (Mazoyer et Roudart, 2002), mais sont aujourd'hui le plus souvent limités aux zones dites "intermédiaires 》, à la lisière des grandes plaines cultivées et des zones plus vallonnées dédiées à l'élevage. Les évolutions profondes de ces dernières décennies ont contribué à une spécialisation des exploitations principalement vers des systèmes de grandes cultures (Hirschler et al., 2019). Dans le contexte de la transition agroécologique, on observe un regain d'intérêt pour la polyculture-élevage, à différentes échelles (exploitation et territoire), pour répondre aux enjeux de multi-performance de l'agriculture (Kremen et al., 2012; Lemaire et al., 2014; Ryschawy et al., 2017). Bien que beaucoup d'études aient analysé les intérêts des systèmes de polyculture-élevage, peu d'entre elles se sont interrogées sur leur avenir. Billen et al. (2019) comparent un scénario tendanciel de spécialisation territoriale à un scénario de diversification des territoires et mettent en évidence que ce dernier, bien que moins productif, permettrait quand même de nourrir la population avec de moindres impacts environnementaux. Des scénarios prospectifs ont été créés pour étudier le changement climatique (Carpenter et Pingali, 2005), la consommation énergétique de l'agriculture française (Vert et Portet, 2010) ou les émissions de gaz à effet de serre (Mosnier et al., 2017b; Vidalenc et al., 2013). Cependant, la place de la polyculture-élevage dans ces scénarios n'a pas été abordée ou très indirectement.

Le projet Casdar RED-SPyCE (Résilience, Efficacité, Durabilité des Systèmes de Polyculture-Élevage) a eu pour principal objectif de constituer une base de connaissances, de pratiques et de références technico-économiques pour améliorer les performances de la polyculture-élevage et contribuer à son maintien. Dans ce cadre, l'étude prospective présentée ici a pour objectif d'imaginer comment pourraient évoluer les systèmes de polyculture-élevage à l'avenir: quelles formes vont-ils prendre? Quelle place vont-ils avoir? Quelles sont les conditions nécessaires à leur développement ?

L'étude a été menée entre 2017 et 2018 dans quatre régions françaises: ex-Midi-Pyrénées, Normandie, ex-Pays de la Loire, ex-Lorraine. Pour ces quatre régions, les résultats de deux approches sont présentés : des ateliers participatifs dans chaque région d'étude et des simulations micro-économiques.

\section{Méthode}

\subsection{Présentation générale de la méthode}

La méthode retenue (Fig. 1) alterne des analyses quantitatives réalisées au bureau (Steinmetz et Mosnier, 2019) et des ateliers participatifs (trois réunions) centrés sur l'analyse du passé puis sur une scénarisation du futur. Cet article présente plus particulièrement la dernière étape, qui consistait, via la modélisation puis les travaux du groupe d'experts (réunion 3), à imaginer comment pourrait évoluer la polyculture-élevage dans les différents scénarios. L'approche par scénario permet d'explorer un futur incertain où chaque scénario décrit des futurs états de la région ou du monde dans leurs différentes dimensions qui doivent être cohérents, plausibles mais pas forcément probables (Abildtrup et al., 2006; Audsley et al., 2006). Contrairement à d'autres travaux de prospective ou au cadre proposé par Miller (2018), les trames de ces scénarios n'ont pas été créées lors des ateliers participatifs, mais reprises en grande partie de l'étude CLIMAGRI (Cascailh et al., 2015) afin de gagner du temps. Ces trames ont été approfondies par les experts du projet Red Spyce pour les trois scénarios: S1 «Ultra-libéralisation et mondialisation galopante», S2 «Économie territoriale et recentrage sur la qualité » et S3 «Transition agroécologique et énergétique» (Tab. 1).

\subsection{Le déroulé de la réunion d'experts sur l'évolution des systèmes de polyculture-élevage}

Le groupe d'animation national (les auteurs de ce papier) a défini le déroulé de la réunion d'experts. Quatre groupes d'experts régionaux ont été constitués, en ciblant prioritairement les acteurs considérés comme incontournables (centres de gestion, recherche et enseignement, chambres d'agriculture, coopératives agricoles, instituts techniques agricoles, acteurs publics régionaux, agence de l'eau). En Lorraine, huit personnes ont participé à cette troisième réunion avec des profils correspondant un peu plus à des acteurs économiques; en Midi-Pyrénées, 21 avec des profils un peu plus orientés vers l'élevage; en Normandie 5 et en Pays de la Loire 7 avec une composante environnementale plus forte (comptes rendus disponibles dans les données supplémentaires en annexe). Dans l'ensemble des régions, $70 \%$ des participants à cette dernière réunion avaient déjà participé au moins à un des groupes précédents, assurant ainsi une certaine continuité dans le travail des experts. Lors de cette réunion, les scénarios retravaillés pendant la réunion 2 (Fig. 1) ont été présentés, ainsi que les matrices de transition qui schématisent les mouvements des exploitations selon leurs orientations de production sur la période 2007-2014 (Hirschler et al., 2019). Les experts ont alors eu pour mission de projeter ces matrices de transition pour les scénarios futurs. Les résultats de simulations réalisées avec le modèle Orfee ont été présentés. Il s'en est suivi une discussion générale, puis une discussion sur les conditions nécessaires au développement de la polyculture-élevage dans chaque scénario. 


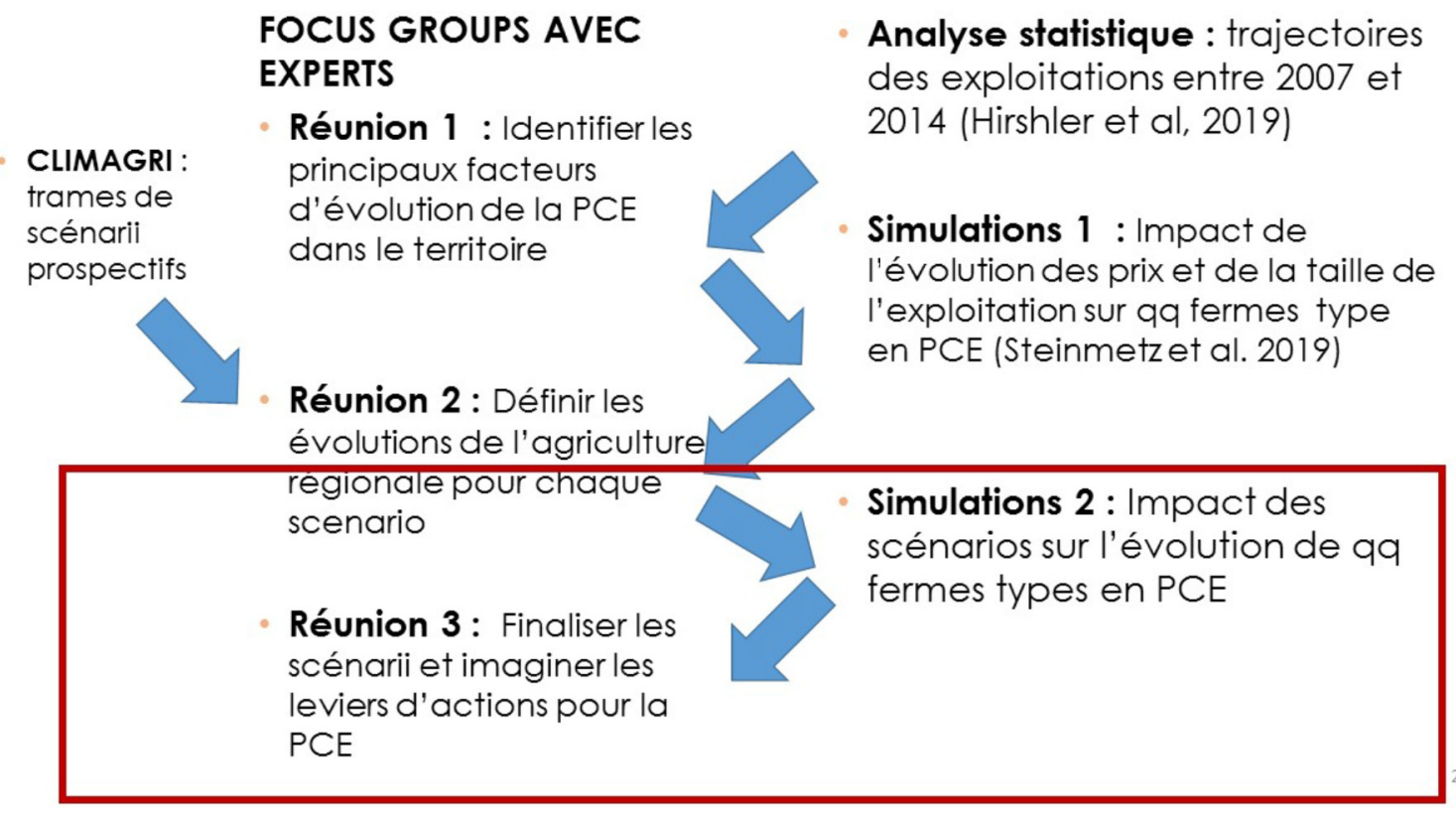

Fig. 1. Les différentes étapes de l'étude prospective.

Fig. 1. The different stages of the prospective study.

\subsection{Les simulations réalisées à partir du modèle Orfee}

\subsubsection{Le modèle}

Le modèle de simulation utilisé - Orfee - est un modèle d'optimisation bioéconomique (Fig. 2) simulant le fonctionnement d'une exploitation agricole en régime de croisière (Mosnier et al., 2017a). Ce modèle a été choisi car il permet de simuler les effets de modifications du contexte politique, économique, technique et structurel sur les choix de production des agriculteurs et de calculer un certain nombre d'indicateurs de performances économiques, sociales et environnementales. L'optimisation est économique, mais les contraintes implémentées via des cahiers des charges et les incitations environnementales simulées via des taxes ou des subventions peuvent infléchir les systèmes vers des systèmes plus respectueux de l'environnement et plus en phase avec les exigences des consommateurs.

Le modèle optimise les choix de production, notamment de taille du troupeau et de bâtiments, d'assolement, de rations des animaux, de main-d'œuvre et de matériel utilisé. Différentes interactions entre les cultures et le bétail ont été prises en compte. L'introduction de cultures fourragères et de prairies temporaires permet une rotation plus longue des cultures, qui nécessitent ainsi moins de produits phytosanitaires (environ $-30 \%$ si ces pratiques sont aussi associées à une baisse de rendement d'environ $6 \%$ ) et d'engrais. La destruction des prairies fournit 50 à $100 \mathrm{~kg}$ d'azote $(\mathrm{N}) /$ ha à la culture suivante (COMIFER, 2013). Dix à $20 \%$ de la teneur en azote du fumier, $80 \%$ de sa teneur en phosphore et toute sa teneur en potassium sont utilisables directement par les plantes. Une partie des éléments fertilisants restants seront disponibles pour les plantes sur le long terme grâce à la minéralisation de la matière organique (environ $10 \mathrm{~kg} \mathrm{~N} /$ ha/an supplémentaire avec des apports de fumiers réguliers). La récolte de la paille utilisée pour la litière animale plutôt que son enfouissement réduit les besoins en azote des cultures de $10 \mathrm{~kg} / \mathrm{ha} / \mathrm{an}$, car la paille, qui a un ratio carbone sur azote élevé, mobilise l'azote présent dans le sol pour permettre sa décomposition. Les régimes alimentaires définis par le modèle doivent répondre aux besoins en protéines et en énergie des animaux et correspondre à leur capacité d'ingestion (INRA, 2007). Les besoins en main-d'œuvre englobent la gestion des cultures (temps pour effectuer les différentes opérations de culture avec les machines appartenant à l'exploitant sélectionné), des troupeaux (reproduction, traite, alimentation et litière, pâturage, etc.) et de l'exploitation dans son ensemble (proportionnelle à la taille de l'exploitation). Pour estimer le nombre d'unités de travail, nous avons supposé qu'une unité de travail associée pouvait travailler au maximum $48 \mathrm{~h}$ par semaine, 49 semaines par an et au maximum $255 \mathrm{~h} / \mathrm{mois}$, et qu'un travailleur salarié pouvait travailler $1645 \mathrm{~h} / \mathrm{an}$.

\subsubsection{Les cas types et scénarios}

Quatre cas types en polyculture-élevage, issus du référentiel INOSYS-Réseau d'élevage, ont été retenus: un système naisseur-engraisseur de bovins sur le plateau lorrain (CVGS4, «cultures et viande sur 250 ha, en zone de polyculture », 2015), un système naisseur de bovins dans les coteaux secs de Midi-Pyrénées (502.1, «Naisseur Blonde d'Aquitaine en coteaux secs avec grandes cultures », 2014), un système bovin laitier dans la Seine maritime en Normandie (6, «Polyculteur laitier de moyenne dimension, 2016) et un système bovins laitiers dans les Pays de la Loire (2B, « lait spécialisé silo ferme-potentiel moyen », 2015). L'assolement, la taille du troupeau et les itinéraires techniques ont été optimisés pour chaque scénario, mais le type d'animaux produits (bovin lait, bovin viande naisseur ou bovin viande naisseur-engraisseur) est fixé, de sorte que ce sont les évolutions 


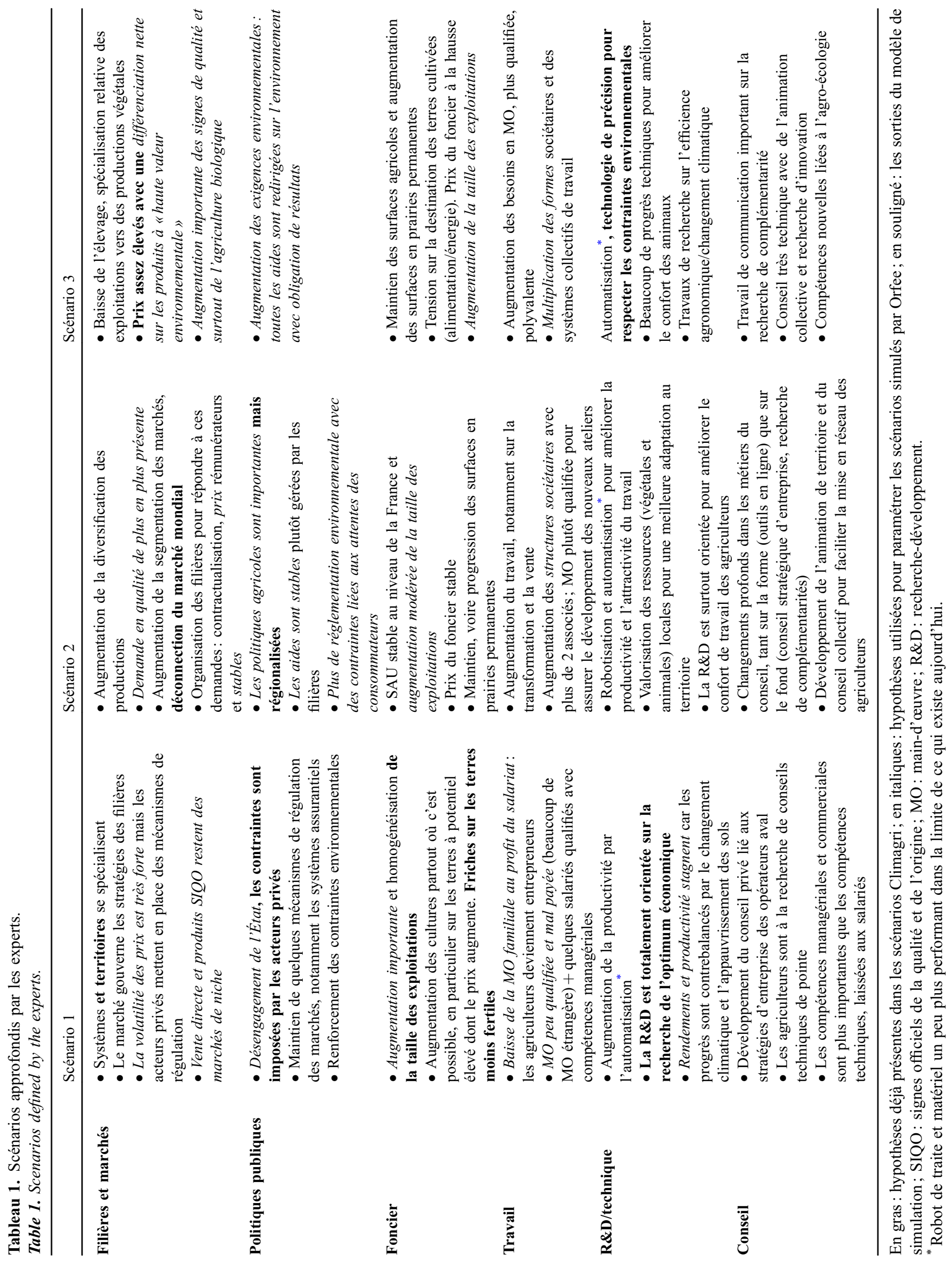




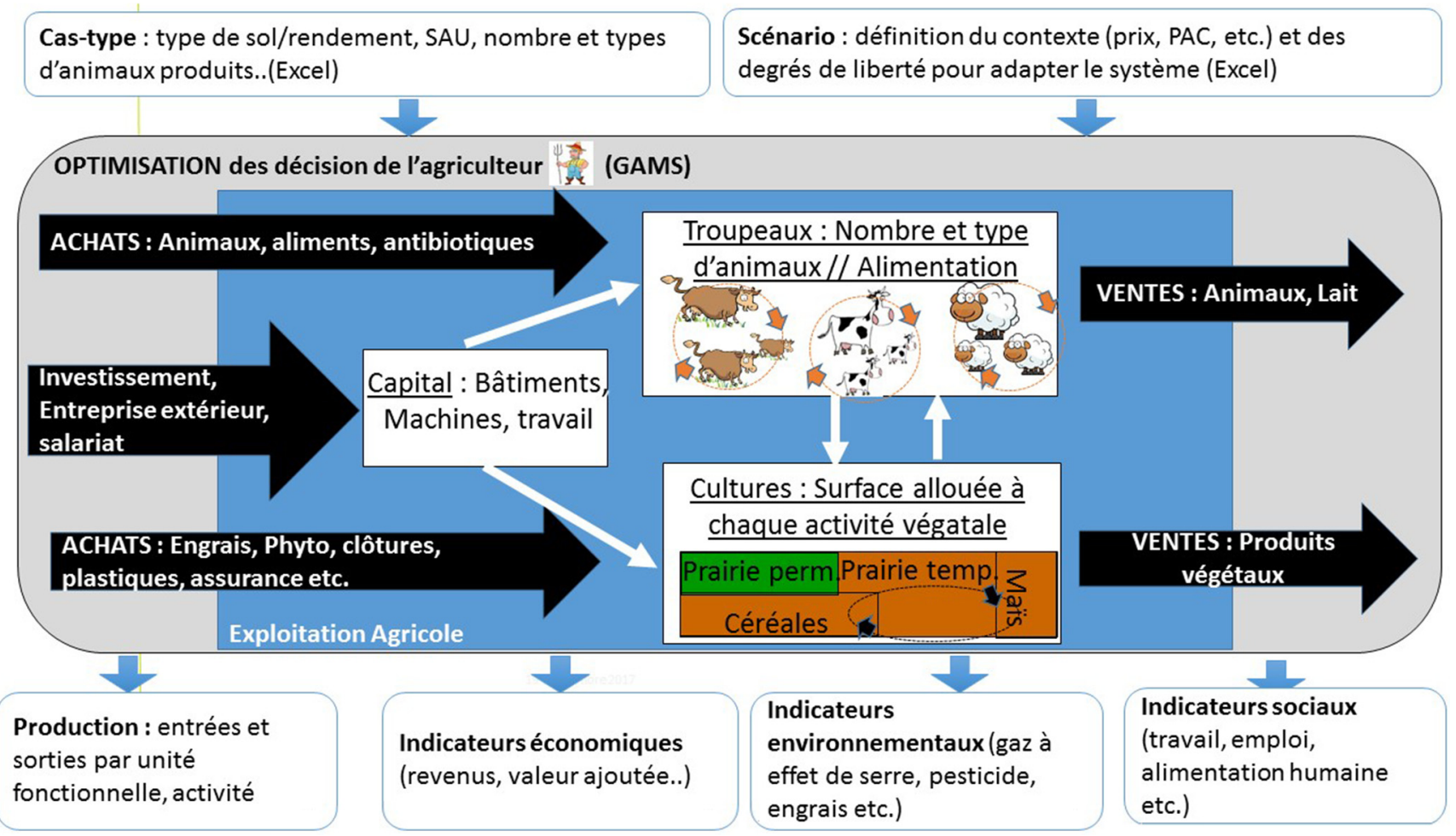

Fig. 2. Représentation du fonctionnement du modèle Orfee.

Fig. 2. Representation of the Orfee model.

de la taille du troupeau, des surfaces, du type de cultures et des modes de production qui sont analysées, et non les transitions d'un type de production animale à un autre. Dans les matrices de transition réalisées par les experts, cela correspond aux passages entre un système spécialisé en élevage, un système de polyculture-élevage associant ce type d'élevage et des grandes cultures, et un système spécialisé en grandes cultures.

Ces cas types ont été projetés en simulant l'impact des éléments clés de chaque scénario (Tab. 2), suivant la même démarche que Mosnier et al. (2017b). Dans le S1 (libéral), les aides directes et les politiques de soutien du revenu sont supprimées. Ce scénario mentionne que certaines politiques environnementales sont maintenues. Néanmoins, afin de contraster les scénarios, nous ne les avons pas considérées. Du S2 (territorial), nous avons conservé l'idée que les aides seraient stables, mais qu'il y aurait davantage de réglementation environnementale et des contraintes supplémentaires liées aux attentes des consommateurs. Les aides actuelles ont été conservées, mais avec des exigences de qualité : animaux alimentés essentiellement à partir d'herbe et cultures gérées de façon intégrée (avec des rotations longues permettant de réduire l'utilisation d'intrants). Pour le S3 (environnement), la lutte contre le réchauffement climatique est au cœur des politiques publiques. Les aides du premier pilier ont été supprimées, une taxe sur les émissions de carbone et une subvention sur son stockage de $40 € / \mathrm{t} \mathrm{CO}_{2}$ équivalent ont été introduites. La taxe sur les émissions augmente les coûts de production et par conséquent les prix (Mosnier et al., 2019). Les prix n'ayant pas été modifiés dans nos scénarios, le niveau de taxe retenu est en deçà du prix plafond de $100 € / \mathrm{t}$ de carbone envisagé à l'horizon 2030 dans le rapport de Canfin et al. (2016).

Concernant les prix moyens, nous avons utilisé ceux de la période 2010-2018, que nous avons fait varier selon les projections de la Commission européenne (European Commission, 2017) et de l'Agence internationale de l'énergie (ADEME, 2012). Nous ne les avons pas fait varier entre les scénarios (excepté pour S3 qui vend ses produits en agriculture biologique). Les experts se sont exprimés en faveur de prix à la hausse pour les scénarios 2 et 3 , mais peu se sont risqués à une quantification et ces estimations étaient divergentes. Les auteurs ont fait le choix de conserver les mêmes niveaux de prix pour les simulations et de discuter les résultats. Concernant la variabilité des prix, des hypothèses contrastées ont été faites par les auteurs, à savoir un doublement de la variabilité des prix dans le S1 dû aux spéculations sur les marchés internationaux, une stagnation dans le S3 et une division par deux dans le S2, qui met en place davantage de régulation des prix et de mécanismes de contrôle des importations et exportations aux frontières (Tab. 2). Pour cela, les variations interannuelles des prix ont été modifiées: un doublement de la variabilité implique par exemple que l'augmentation de $12 \%$ du prix du lait observée en 2014, par rapport à la période de référence de 2010-2018, passe à $24 \%$ et que la baisse de $11 \%$ observée en 2016 passe à $22 \%$.

La taille des exploitations augmente dans tous les scénarios, mais plus fortement dans le S1. Nous avons retenu un doublement de la taille des exploitations associé à la baisse du nombre d'exploitations pour S1, une augmentation de $30 \%$ pour S3 et de seulement $10 \%$ pour S2. 
Tableau 2. Principales hypothèses retenues pour les scénarios Orfee.

Table 2. Main assumptions used for the Orfee scenarios.

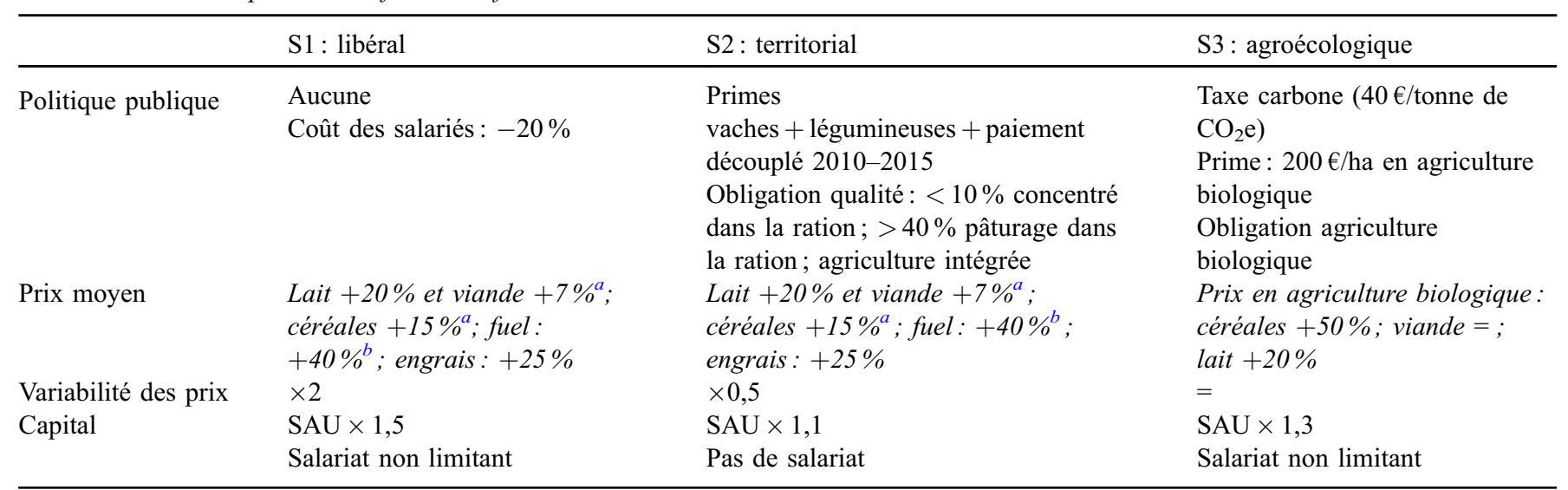

En italiques: hypothèses non basées sur les scénarios approfondis par les experts.

${ }^{a}$ Projection commission européenne.

${ }^{\mathrm{b}}$ Agence internationale de l'énergie. Variations par rapport à la moyenne des prix observés sur la période 2010-2015.

\subsection{Résultats des simulations}

L'évolution de la polyculture-élevage est évaluée à travers quatre indicateurs : la part des ventes animales par rapport aux ventes totales (les intra-consommations de céréales sont considérées comme des ventes) permet d'estimer le poids économique des deux activités, la part de la surface intraconsommée par le troupeau (surface fourragère et grains) dans la surface agricole utile (SAU) indique le poids de l'élevage en terme de surface. Des valeurs proches de $0 \%$ ou de $100 \%$ reflètent des systèmes spécialisés, des valeurs autour de $50 \%$ témoignent de systèmes où cultures de vente et élevage sont tous deux importants. Une autonomie alimentaire élevée du troupeau et une faible consommation l'azote minérale par unité de SAU sont des signes d'une bonne intégration entre cultures et élevage.

Pour le S1, le poids de l'élevage laitier est proche de la période de référence. L'autonomie alimentaire est plus importante malgré un niveau de chargement plus élevé, grâce à l'intensification des surfaces (Tab. 3). La polyculture-élevage avec des vaches allaitantes tend à disparaître. Dans ce scénario, l'augmentation des prix de la viande ne compense pas la suppression des aides couplées pour les vaches allaitantes. Dans le S2, le poids de la production laitière et le niveau de chargement sont réduits, afin de respecter les contraintes de pâturage minimum et de part maximale des concentrés. C'est le scénario pour lequel le poids de l'élevage laitier est le plus proche de celui des grandes cultures. L'achat d'azote diminue et l'autonomie reste élevée. L'élevage allaitant est maintenu à un niveau proche de la situation initiale, grâce notamment au maintien des aides couplées. Dans le S3, bien qu'il y ait une taxe sur les émissions de gaz à effet de serre, l'interdiction des fertilisants de synthèse et la nécessité d'avoir des rotations longues sont favorables à l'élevage et aux prairies, mais avec des niveaux de chargement plus faibles. Pour les systèmes de polyculture-élevage avec des vaches allaitantes, la part des grandes cultures est à peu près équivalente à celle de l'élevage.
Plusieurs indicateurs ont été retenus pour mesurer la durabilité des exploitations. Le S1 apparaît le plus favorable pour la dimension économique, excepté pour la variabilité des revenus, ce qui s'explique par le faible niveau de contrainte qui pèse sur le système de production, alors que les prix diffèrent peu des autres scénarios. La dimension sociale est appréhendée par la contribution à l'emploi local mesurée par les heures de travail générées au mètre carré, et par la contribution à l'alimentation mesurée par la production de protéines à l'hectare et par des indicateurs de qualité de la viande produite. Ces indicateurs sont meilleurs dans le S2. Le S3 présente de meilleurs indicateurs environnementaux grâce à l'absence d'utilisation de pesticides et de fertilisants de synthèse. Les émissions de gaz à effet de serre (nettes de stockage) par hectare ne sont pas toujours les plus faibles, cela dépend essentiellement du niveau de chargement animal simulé pour chaque scénario.

\subsection{La scénarisation issue des focus groups}

Les matrices de transition proposées par les experts de Midi-Pyrénées sont présentées dans la figure 3. Les évolutions anticipées par les experts des autres régions sont uniquement intégrées dans le texte qui suit. Pour les experts de l'ensemble des régions, le $\mathrm{S} 1$ est défavorable à la polyculture-élevage et à l'agriculture en général (Fig. 3). Cela se traduit par une baisse considérable du nombre d'exploitations agricoles, en particulier en élevage. Pour les élevages ayant des animaux de race à viande, on assiste à une baisse très importante des élevages spécialisés. Une désintensification des systèmes naisseurs est projetée en Lorraine et en Midi-Pyrénées, avec la mise en place d'ateliers d'engraissement très intensifs. Les exploitations de polyculture-élevage laitières diminuent drastiquement, voire disparaissent, et hormis en Pays de la Loire, seules quelques exploitations spécialisées se maintiennent au prix d'agrandissements importants et d'une forte spécialisation pour optimiser les coûts. Beaucoup de systèmes de polyculture-élevage se transforment en systèmes de grandes cultures spécialisés. 
Tableau 3. Évolution des exploitations et de leur durabilité selon les scénarios simulés par le modèle Orfee.

Table 3. Evolution of farms and their sustainability according to the simulated scenarios by the Orfee model.

\begin{tabular}{|c|c|c|c|c|c|c|c|c|c|c|c|c|c|c|c|c|}
\hline & \multicolumn{4}{|c|}{ Ex-Pays de la Loire } & \multicolumn{4}{|c|}{ Normandie } & \multicolumn{4}{|c|}{ Ex-Lorraine } & \multicolumn{4}{|c|}{ Ex-Midi-Pyrénées } \\
\hline & Base & $\mathrm{S} 1$ & $\mathrm{~S} 2$ & $\mathrm{~S} 3$ & Base & $\mathrm{S} 1$ & $\mathrm{~S} 2$ & S3 & Base & $\mathrm{S} 1$ & $\mathrm{~S} 2$ & S3 & Base & $\mathrm{S} 1$ & $\mathrm{~S} 2$ & $\mathrm{~S} 3$ \\
\hline \multicolumn{17}{|l|}{ Production } \\
\hline SAU (ha) & 76 & 114 & 84 & 99 & 190 & 285 & 209 & 247 & 250 & 375 & 275 & 325 & 105 & 158 & 116 & 137 \\
\hline Prairies (\%) & 57 & 6 & 34 & 17 & 24 & 13 & 20 & 56 & 24 & 16 & 25 & 43 & 43 & 19 & 50 & 55 \\
\hline Maïs ensilage (\%) & 29 & 27 & 14 & 5 & 16 & 13 & 12 & 12 & 4 & & 1 & 1 & & & & \\
\hline Légumineuses (\%) & 0 & 27 & 7 & 10 & 8 & 13 & 19 & 49 & 2 & 0 & 11 & 0 & 10 & 1 & 0 & 0 \\
\hline Céréales et oléagineux (\%) & 14 & 40 & 45 & 60 & 52 & 61 & 49 & 17 & 70 & 84 & 47 & 49 & 40 & 80 & 47 & 45 \\
\hline Taille du troupeau (UGB) & 96 & 143 & 54 & 46 & 144 & 247 & 152 & 209 & 97 & 0 & 87 & 142 & 98 & 41 & 69 & 76 \\
\hline $\mathrm{UGB} / \mathrm{ha} \mathrm{SAU}$ & 1 & 1,3 & 1,3 & 0,6 & 0,5 & 0,8 & 0,9 & 0,7 & 0,8 & 0 & 0,3 & 0,4 & 0,9 & 0,3 & 0,6 & 0,6 \\
\hline Chargement (UGB/ha SFP) & 1,5 & 2,1 & 1,2 & 1,5 & 1,8 & 2,2 & 1,9 & 1,3 & 1,3 & 0 & 1,2 & 1,0 & 1,6 & 1,3 & 1,2 & 1,0 \\
\hline Production de lait par vache (1000L/VL) & 7,4 & 7,4 & 6,1 & 7,1 & 8,3 & 8,3 & 5,9 & 8,3 & & & & & & & & \\
\hline $\mathrm{kg}$ viande/UGB & 151 & 151 & 134 & 151 & 174 & 174 & 153 & 173 & 376 & 0 & 379 & 377 & 322 & 328 & 326 & 324 \\
\hline Travail (UMO) & 2,0 & 4,0 & 1,7 & 1,0 & 2,3 & 6,4 & 3,2 & 4,2 & 1,6 & 1,7 & 1,4 & 2,1 & 1,2 & 1,0 & 0,9 & 1,0 \\
\hline \multicolumn{17}{|l|}{ Polyculture-élevage } \\
\hline $\begin{array}{l}\text { Part des ventes animales }(\% \text { ventes } \\
\text { totales)* }\end{array}$ & 95 & 86 & 72 & 59 & 63 & 57 & 57 & 93 & 28 & 0 & 25 & 37 & 68 & 15 & 49 & 50 \\
\hline Surface intra-consommée ( $\% \mathrm{SAU})$ & 86 & 80 & 58 & 32 & 43 & 47 & 42 & 100 & 32 & & 29 & 43 & 58 & 24 & 52 & 55 \\
\hline Autonomie alimentaire $(\%)^{* *}$ & 66 & 98 & 97 & 100 & 72 & 93 & 97 & 99 & 73 & & 96 & 95 & 89 & 99 & 98 & 99 \\
\hline Azote sur grande culture $(€ / \mathrm{ha})$ & 106 & 136 & 99 & 0 & 172 & 202 & 156 & 0 & 159 & 225 & 138 & 0 & 62 & 212 & 127 & 0 \\
\hline \multicolumn{17}{|l|}{ Durabilité économique } \\
\hline Résultat courant ( $\mathrm{k} € /$ travailleur) & 18 & 81 & 48 & 48 & 66 & 289 & 74 & 103 & 48 & 183 & 74 & 28 & 21 & 104 & 61 & 47 \\
\hline $\begin{array}{l}\mathrm{RC} / \text { travailleur hors aide (prix } \\
\text { conventionnel 2010) }\end{array}$ & 10 & 21 & 10 & -6 & 47 & 43 & 32 & 9 & 14 & 45 & 18 & -28 & -7 & 10 & -7 & -24 \\
\hline $\begin{array}{l}\text { Stabilité du revenu (écart type } \mathrm{RC} \\
\mathrm{k} \in \text { /travailleur) }\end{array}$ & 4 & 33 & 3 & 11 & 10 & 66 & 5 & 11 & 12 & 88 & 9 & 12 & 4 & 42 & 5 & 9 \\
\hline Dépendance aux aides (Subv \% RC) & 72 & 0 & 43 & -16 & 32 & 0 & 23 & 24 & 78 & 0 & 68 & 77 & 197 & 0 & 65 & 42 \\
\hline \multicolumn{17}{|l|}{ Durabilité sociale } \\
\hline Emploi (heures de travail $/ \mathrm{m}^{2}$ ) & 0,6 & 0,5 & 0,4 & 0,2 & 0,3 & 0,4 & 0,4 & 0,4 & 0,2 & 0,1 & 0,1 & 0,2 & 0,3 & 0,1 & 0,2 & 0,2 \\
\hline $\begin{array}{l}\text { Qualité animale (\% d'herbe pâturée sur } \\
\text { l'année) }\end{array}$ & 11 & 1 & 23 & 11 & 13 & 12 & 26 & 13 & 23 & & 42 & 37 & 20 & 45 & 44 & 40 \\
\hline Concentré (kg/UGB) & 1065 & 1435 & 573 & 885 & 1297 & 1408 & 470 & 873 & 529 & & 489 & 764 & 484 & 1263 & 285 & 486 \\
\hline $\begin{array}{l}\text { Production de protéines alimentaires } \\
(\mathrm{kg} / \mathrm{ha})\end{array}$ & 284 & 320 & 305 & 206 & 466 & 342 & 547 & 198 & 231 & 287 & 298 & 123 & 77 & 259 & 232 & 99 \\
\hline \multicolumn{17}{|l|}{ Durabilité environnementale } \\
\hline Gaz à effet de serre (émission nette/ha) & 6263 & 6509 & 2655 & 2035 & 4811 & 5332 & 3759 & 4581 & 1766 & 458 & 1094 & 1149 & 3384 & 1287 & 1757 & 1071 \\
\hline Pesticides ( $€ /$ ha) & 34 & 87 & 61 & 0 & 102 & 127 & 87 & 0 & 125 & 176 & 72 & 0 & 58 & 207 & 79 & 0 \\
\hline Fertilisants minéraux ( $€ /$ ha $)$ & 39 & 63 & 50 & 0 & 123 & 148 & 109 & 0 & 132 & 194 & 114 & 0 & 47 & 173 & 68 & 0 \\
\hline
\end{tabular}

«Base» : simulations pour le contexte 2010-2018 pour un assolement et une taille de troupeau identique au cas type de référence. En vert : situation la plus favorable; en orange: situation la moins favorable; *évaluée en euros 2010; **autonomie en énergie (unités fourragères).

Les attentes croissantes des consommateurs pour des produits locaux dans le S2 sont favorables au maintien des systèmes de polyculture-élevage, en particulier les exploitations non laitières qui se diversifient encore plus avec des ateliers de monogastriques ou de petits ruminants, ou côté végétal avec le développement de légumineuses, maraîchage, agroforesterie, etc. Les experts envisagent une baisse du nombre d'exploitations, mais inférieure à la tendance actuelle. La condition pour que les systèmes mixtes se pérennisent dans ce scénario est l'émergence de démarches collectives fortes dans les territoires, portées par les agriculteurs ou par les filières. Sont notamment nécessaires des outils collectifs pour gérer la transformation, le transport et la vente en filières courtes, mais également des collectifs de travail. Ces démarches sont soutenues par les pouvoirs publics et en particulier par les régions qui s'engagent fortement dans les territoires ruraux. Elles peuvent également être engagées avec l'appui des associations de consommateurs, devenues très actives dans ce scénario.

Le S3 est le moins consensuel entre les régions. En Lorraine et en Normandie (S3A), les experts voient dans l'orientation agroécologique du scénario une opportunité pour la polyculture-élevage. Les exploitations de grandes cultures réintroduisent de l'élevage, notamment pour valoriser les terres les moins productives. Les élevages spécialisés, eux, tendent à augmenter leur part en cultures pour chercher l'autonomie alimentaire, celle-ci étant bien valorisée auprès des consommateurs. En Midi-Pyrénées et en Normandie (S3B), la baisse de la consommation de viande et le souci de réduire les gaz à effet de serre sont vus comme des facteurs concourant à la diminution globale de l'élevage. Les modèles d'élevage qui persistent sont plutôt très extensifs. L'élevage laitier migre soit vers de l'allaitant, soit vers des grandes cultures. Les exploitations laitières qui se maintiennent sont 
a) Scénario 1 : « Libéral »

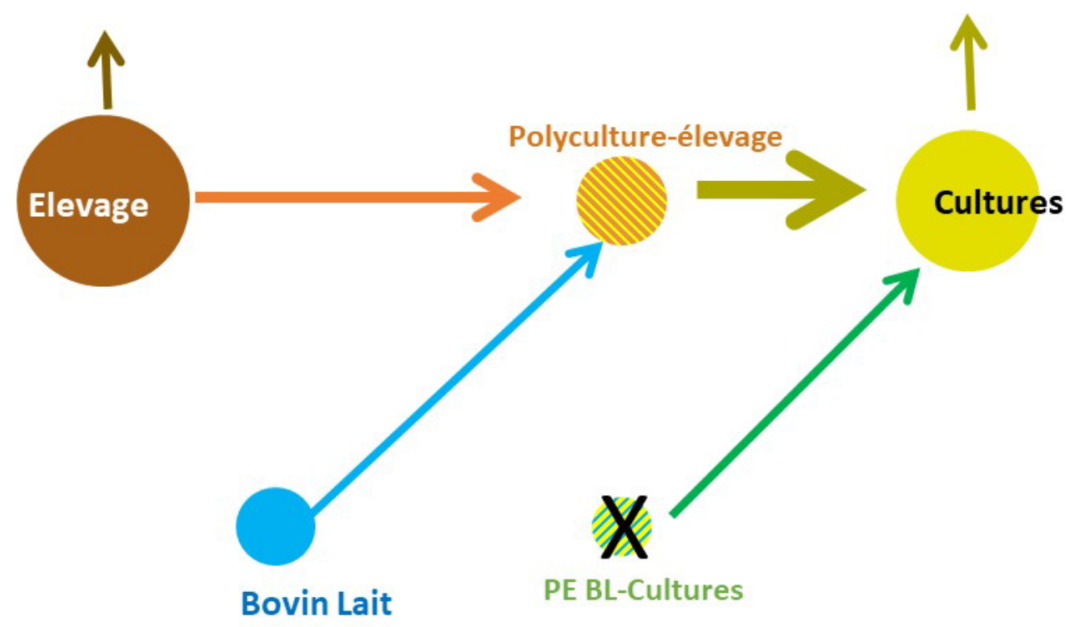

b) Scénario 2 : "territorial"

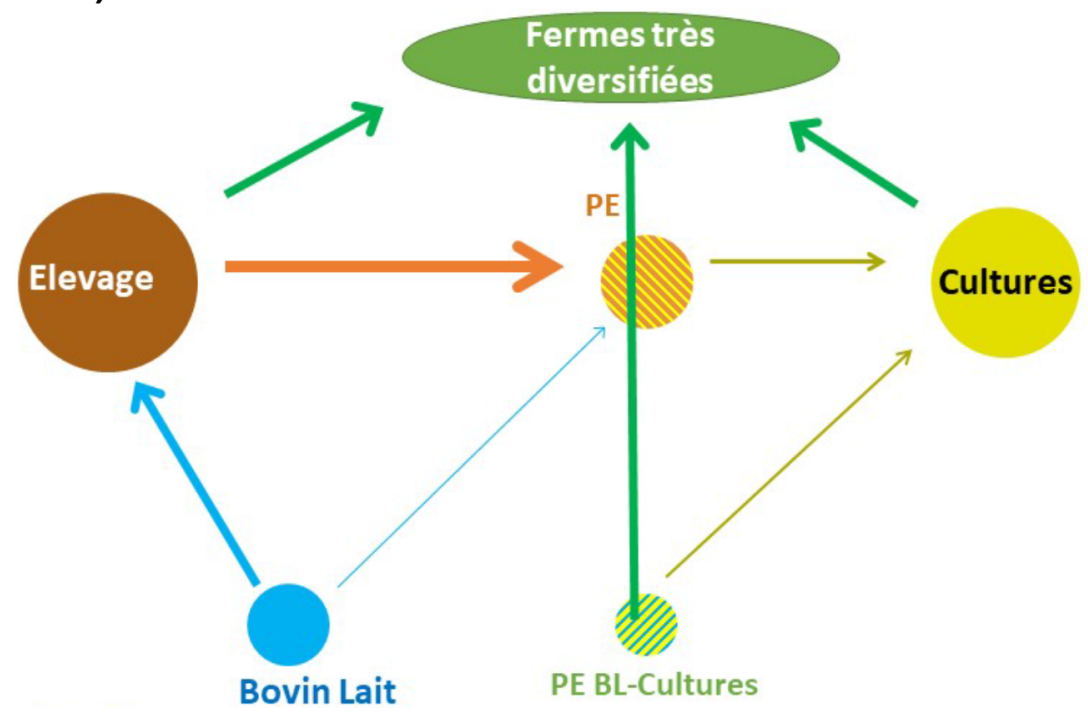

c) Scénario 3 : “Agro-écologique

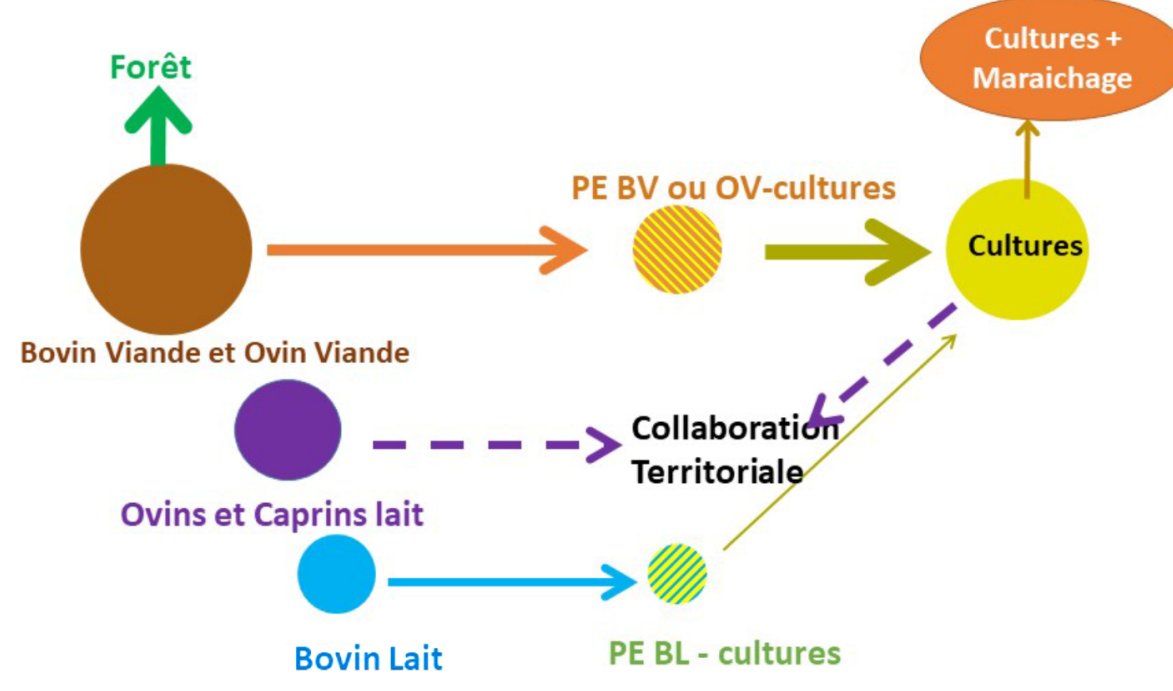

Fig. 3. Matrice de transition des fermes d'élevage réalisée par le groupe d'experts de la région Midi-Pyrénées.

Fig. 3. Transition matrix of livestock farms carried out by the Midi-Pyrénées group of experts. 
plutôt des exploitations de polyculture-élevage très autonomes, de grande taille, et inscrites dans des filières bien valorisées. Les terres peu productives sont réorientées vers la forêt, bien valorisée dans ce scénario. Dans cette orientation, les politiques publiques encouragent plutôt la polycultureélevage de territoire en soutenant les échanges entre céréaliers et éleveurs. Un autre élément constant entre les régions est le développement important des cultures de légumineuses, à la fois pour l'alimentation animale en recherche d'autonomie et pour l'alimentation humaine, en compensation de la baisse des protéines animales.

\section{Discussion}

\subsection{Devenir de la polyculture-élevage selon les scénarios et les méthodes utilisées}

Les résultats des ateliers participatifs et des simulations de l'évolution d'exploitations s'accordent sur le fait que le S1 (libéral) va vers une réduction de l'élevage de bovins viande, qui s'extensifie (au moins pour la phase allaitante) au profit des grandes cultures. Les experts prédisent une réduction de la production laitière. Il n'en est rien dans les simulations. Cela peut être relié à des hypothèses qui n'ont pas été bien partagées ou qui n'ont pas eu le même poids entre les deux démarches : les simulations ont considéré une augmentation des prix et une main-d'œuvre disponible peu qualifiée, favorisant le maintien de la production laitière. Peut-être les experts pensaient-ils que les prix seraient plus bas que ceux simulés ou que l'agriculture française ne serait pas compétitive avec l'agriculture mondiale.

Experts et résultats de simulations s'accordent sur le fait que le S2 (territorial) est le plus favorable à des systèmes de polyculture-élevage, avec une importance accrue des légumineuses. Les experts envisagent une augmentation du nombre d'ateliers dans l'exploitation, qui n'a pas pu être simulée avec le modèle. Les simulations indiquent que les niveaux de revenus sont intermédiaires. Ce résultat est lié aux hypothèses d'augmentation des prix agricoles et des niveaux de soutiens publics, ce qui signifie que consommateurs et/ou citoyens doivent être prêts à consacrer une part plus importante de leur revenu pour l'alimentation. Concernant l'hypothèse de stabilité des prix, elle ne peut être vraie que si l'agriculture locale n'est pas complètement déconnectée du marché international pour tamponner les aléas de production et qu'il y a des stocks tampons importants.

Le S3 (environnement) voit une baisse de l'élevage, mais une intégration plus forte entre élevage et cultures. La fourniture d'azote est cruciale en agriculture biologique (Seufert et Ramankutty, 2017). Le maintien de l'élevage s'explique ici par la nécessité de fertiliser les terres, soit directement via les effluents, soit indirectement via les légumineuses fourragères. Cependant, le maintien d'une part importante d'élevage géré de façon extensive, associé au passage en agriculture biologique, induit une baisse de la production de protéines à l'hectare. Cette baisse doit être compensée par l'utilisation de surfaces plus importantes pour nourrir la population humaine, ce qui pose problème pour la biodiversité et le bilan global des gaz à effet de serre (Searchinger et al., 2018; Van Zanten et al., 2018). Les hypothèses fortes de baisse de rendement des légumineuses à grain $(>50 \%)$ en agriculture biologique peuvent expliquer qu'elles soient peu utilisées dans les simulations et que l'élevage soit privilégié.

Les experts imaginent aussi dans ce scénario, ainsi que dans le S2, des interactions plus fortes entre exploitations spécialisées à l'échelle du territoire, qui n'ont pas été testées dans les simulations. Plusieurs projets, passés et en cours, mettent en effet en avant leurs avantages (projet européen Cantogether, PSDR Poeete, SOS-Protéine, etc.).

\subsection{Retour sur la méthode}

Les résultats issus des différentes réunions d'experts régionaux et du modèle ont permis de donner une vision différente et complémentaire de la place et de la forme de la polyculture-élevage à l'avenir. Les résultats du modèle ont suscité des interrogations et des discussions, notamment concernant les résultats du scénario «libéral». Nous n'avons pas pu mesurer à quel point ces discussions avaient modifié la vision des choses de ces experts, dans la mesure où nous n'avions pas assez de temps pour leur faire «revoir» leur vision de la polyculture-élevage suite aux discussions. Certaines remarques des experts ont été prises en compte pour améliorer les hypothèses retenues dans les simulations, notamment les niveaux d'intensification possibles dans les différents scénarios. Pour plus d'interactions entre simulations et ateliers participatifs, plusieurs sessions de travail auraient été nécessaires pour modifier pas à pas les scénarios. Le nombre et la durée des ateliers participatifs étant très limités, ce processus a été complété par des discussions lors des phases de restitution du projet. Concernant le type de modélisation retenu, son intérêt est qu'il permet de projeter des évolutions de système de production selon des hypothèses explicites et de prendre en compte simultanément des aspects économiques, structurels, techniques ou environnementaux. Ses limites sont d'une part la difficulté de bien faire comprendre tous les processus en jeu sans rentrer dans la complexité du modèle, et d'autre part des futurs possibles qui se limitent aux capacités du modèle.

Concernant le déroulement des ateliers participatifs, dont celui présenté était l'aboutissement, les experts se sont déclarés satisfaits dans toutes les régions. Ils ont apprécié la démarche même si le délai entre les réunions (presque une année entre la deuxième et la troisième) était jugé trop long à l'unanimité, ce qui a altéré la dynamique et la continuité du processus prospectif.

\section{Conclusion}

L'objectif de cet article était de présenter et de comparer les résultats issus des ateliers participatifs et des simulations micro-économiques sur l'évolution des systèmes de polyculture-élevage selon trois scénarios prospectifs: «Ultralibéralisation et mondialisation galopante» (S1), «Économie territoriale et recentrage sur la qualité » (S2) et «Transition agroécologique et énergétique » (S3).

Nous montrons des convergences dans les deux types d'approches, notamment sur la réduction de l'élevage de bovins viande, qui s'extensifie au profit des grandes cultures dans le S1, sur un S2 plus favorable aux systèmes de polyculture-élevage et un S3 qui voit une baisse de l'élevage mais avec une intégration plus forte entre élevage et culture. Il existe également des 
divergences, comme celle de l'évolution de la production laitière dans le S1 et la part de l'élevage dans le S3. Chaque approche permet d'approfondir certaines dimensions: les approches participatives ont permis de creuser la dimension territoriale et d'envisager tous les types de combinaisons élevage-production végétale. La simulation a permis d'analyser les liens de causalités entre changement du contexte de production, évolution des systèmes de production et durabilité de ces derniers.

\section{Matériel supplémentaire}

Réunions du Groupe d'Exerperts Casdar 5521 «RED-SPyCE». Le matériel supplémentaire est disponible sur https://www. cahiersagricultures.fr//10.1051/cagri/2020028/olm.

Remerciements. Nous adressons nos remerciements à l'ensemble des partenaires du projet RED-SPyCE qui ont permis la réalisation de ce travail riche en échanges. Nous remercions également l'ensemble des personnes ayant participé aux groupes d'experts dans les différentes régions et ayant ainsi contribué à la réalisation globale de cette étude. Enfin, nous remercions les relecteurs anonymes qui ont permis d'améliorer cet article.

\section{Références}

Abildtrup J, Audsley E, Fekete-Farkas M, Giupponi C, Gylling M, Rosato P, et al. 2006. Socio-economic scenario development for the assessment of climate change impacts on agricultural land use: a pairwise comparison approach. Environmental Science \& Policy 9 (2): 101-115.

ADEME. 2012. L'exercice de prospective de l'ADEME « Vision 2030-2050 ». Angers, France: ADEME.

Audsley E, Pearn KR, Simota C, Cojocaru G, Koutsidou E, Rounsevell MDA, et al. 2006. What can scenario modelling tell us about future European scale agricultural land use, and what not? Environmental Science \& Policy 9(2):148-162. http://dx.doi.org/ 10.1016/j.envsci.2005.11.008.

Billen G, Le Noë J, Anglade J, Garnier J. 2019. Polyculture-élevage ou hyper-spécialisation territoriale ? Deux scénarios prospectifs du système agro-alimentaire français. Innovations Agronomiques 72: 31-44. https://hal.inrae.fr/hal-02627306.

Canfin P, Grandjean A, Mestrallet G. 2016. Propositions pour des prix du carbone alignés avec l'Accord de Paris. In : Rapport de la mission remis à Ségolène Royal, en sa qualité de présidente de la COP21. https://www.ecologique-solidaire.gouv.fr/sites/default/ files/Rapport\%20Canfin\%20Grandjean\%20Mestrallet.pdf.

Carpenter S, Pingali P. 2005. Millennium ecosystem assessmentscenarios assessment. Washington DC, USA: Island Press.

Cascailh A, Dubosc N, Longueval C, Nedellec J, Tizon A, Vandewalle A. 2015. Étude Climagri région Midi-Pyrénées. Toulouse, France: Chambre Agriculture Midi-Pyrénées.

COMIFER. 2013. Calcul de la fertilisation azotée. p. 159. https://comifer. asso.fr/fr/publications/les-brochures.html (accès : août 2020).

European Commission. 2017. EU Agricultural Outlook for the agricultural markets and income 2017-2030. Bruxelles, Belgique: European Commission.
INRA. 2007. Alimentation des bovins, ovins et caprins : besoins des animaux-valeurs des aliments. Paris: Éditions Quae, 307 p.

Hirschler J, Stark F, Gourlaouen Y, Perrot C, Dubosc N, Ramonteu S. 2019. Évolution des systèmes de polyculture-élevage : une rétrospective statistique 2007-2014. Innovations Agronomiques 72: 193-209. https://doi.org/10.15454/HPTJH1.

Kremen C, Iles A, Bacon CM. 2012. Diversified farming systems: an agroecological, systems-based alternative to modern industrial agriculture. Ecology and Society 17(4): 44. https://doi.org/10.5751/ ES-05103-170444.

Lemaire G, Franzluebbers A, Carvalho PCdF, Dedieu B. 2014. Integrated crop-livestock systems: strategies to achieve synergy between agricultural production and environmental quality. Agriculture, Ecosystems \& Environment 190: 4-8. https://doi. org/10.1016/j.agee.2013.08.009.

Mazoyer M, Roudart L. 2002. Histoire des agricultures du monde: du néolithique à la crise contemporaine, Histoire. Paris: Éditions du Seuil.

Miller R. 2018. Transforming the future. Anticipation in the 21st century. Paris, France: UNESCO and Routledge, 301 p.

Mosnier C, Britz W, Julliere T, De Cara S, Jayet PA, Havlík P, et al. 2019. Greenhouse gas abatement strategies and costs in French dairy production. Journal of Cleaner Production 236: 117589. https://doi.org/10.1016/j.jclepro.2019.07.064.

Mosnier C, Duclos A, Agabriel J, Gac A. 2017a. Orfee: a bioeconomic model to simulate integrated and intensive management of mixed crop-livestock farms and their greenhouse gas emissions. Agricultural Systems 157: 202-215. https://doi.org/10.1016/j. agsy.2017.07.005.

Mosnier C, Duclos A, Agabriel J, Gac A. 2017b. What prospective scenarios for 2035 will be compatible with reduced impact of French beef and dairy farm on climate change? Agricultural Systems 157: 193-201. https://doi.org/10.1016/j.agsy.2017.07.006.

Ryschawy J, Martin G, Moraine M, Duru M, Therond O. 2017. Designing crop-livestock integration at different levels: toward new agroecological models? Nutrient Cycling in Agroecosystems 108: 5-20. https://doi.org/10.1007/s10705-016-9815-9.

Searchinger TD, Wirsenius S, Beringer T, Dumas P. 2018. Assessing the efficiency of changes in land use for mitigating climate change. Nature 564(7735): 249-253. https://doi.org/10.1038/s41586-0180757-z.

Seufert V, Ramankutty N. 2017. Many shades of gray-The contextdependent performance of organic agriculture. Science Advances 3 (3): e1602638. https://doi.org/10.1126/sciadv.1602638.

Steinmetz L, Mosnier C. 2019. Adaptation des systèmes de polycultureélevage aux variations de prix et de taille de l'exploitation : simulation à partir du modèle bioéconomique Orfee. Innovations Agronomiques 72: 77-89. https://doi.org/10.15454/o65nf2.

Vert J, Portet F. 2010. Prospective agriculture énergie 2030. L'agriculture face aux défis énergétiques. Paris, France: Centre d'études et de prospective, SSP, ministère de l'Agriculture, de l'Alimentation, de la Pêche, de la Ruralité et de l'Aménagement du territoire.

Vidalenc E, Meunier L, Topper B. 2013. Visions Ademe 2030-2050. Revue de l'énergie (612): 85-94.

Van Zanten HHE, Herrero M, Van Hal O, Röös E, Muller A, Garnett T, et al. 2018. Defining a land boundary for sustainable livestock consumption. Global Change Biology 24(9): 4185-4194. https:// doi.org/10.1111/gcb.14321.

Citation de l'article : Mosnier C, Dubosc N, Abdouttalib I, Candau D, Carel Y, Chauvat S, Fougy F, Guerre É, Magnin L, Ramonteu S. 2020. Quelles évolutions possibles pour les systèmes de polyculture-élevage? Résultats d'ateliers participatifs et de modélisation dans quatre régions françaises. Cah. Agric. 29: 30 . 